\title{
Preoperative MRI of perianal fistula evaluation and its impact on surgical outcome
}

\author{
Hesham Youssef Algazzar ${ }^{1 *}$, Diaa Bakry Eldib ${ }^{1}$, Mahmoud A. Bahram² and Nasser A. Zaher ${ }^{3}$
}

\begin{abstract}
Background: Perianal fistula (PAF) is an abnormal tract communicating an external cutaneous opening in the perianal region to the anal canal. PAF is one of the common anorectal disorders in surgical practice with high prevalence. The current study aimed to determine the ability of preoperative MRI for preoperative evaluation of perianal fistula.
\end{abstract}

Results: This is a retrospective evaluation of 65 patients with perianal fistula. MRI fistula imaging-related data were revised, and fistula severity was scored using criteria of both local extension of fistulas and active inflammation for a total maximum score of 22. Preoperative MRI could predict the severity of perianal disease with sensitivity, specificity, and accuracy rates of $75 \%, 92 \%$, and $84.6 \%$, respectively. Surgical findings concerning PAF severity correlated significantly with MRI findings. Diffusion-weighted magnetic resonance imaging (DW-MRI) provided high sensitivity and accuracy with 100\% specificity for fistula visualization and highest sensitivity, specificity, and accuracy for detection of cavities $>3 \mathrm{~mm}$ in diameter. DW-MRI provided the highest specificity rate on ROC curve analysis among the three MRI pulse sequences (DW-MRI, short tau inversion recovery (STIR), and T1 post-contrast).

Conclusion: MRI is valuable and accurate for preoperative investigation for PAF evaluation and abscess localization. MRI allowed accurate detection of internal fistula opening and its relation to sphincters. DW-MRI is a valuable sequence with highest diagnostic yield, and its addition to STIR WI improves sensitivity and specificity for determination of fistula activity and extension.

Keywords: Perianal fistula activity, Preoperative MRI, Surgical decision making, MRI pulse sequences

\section{Background}

Perianal fistula (PAF) is an abnormal tract communicating an external cutaneous opening in the perianal region to an internal opening, most often in the anal canal [1]. PAF is one of the common anorectal disorders in surgical practice [2] with high prevalence, which predominantly affects young adult males [3].

Anal glands are situated in the intramuscular plane at the level of the dentate line in the anal canal [4]. The burden of anorectal sepsis is high [5], and persistent infection may spread in circumferential or in axial direction, resulting in different types of fistulas [4] within the

\footnotetext{
* Correspondence: heshamyg@hotmail.com

${ }^{1}$ Radiodiagnosis Department, Faculty of Medicine, Benha University, Benha, Egypt

Full list of author information is available at the end of the article
}

first year of presentation with an abscess [5]. Clinically, the Parks Classification and Perianal Disease Activity Index (PDAI) can be considered as the milestone for classifying patients with PAF and as the gold standard for evaluating its complexity and severity [6].

Effective surgical treatment of PAF is mandatory to prevent its recurrence [7]. However, the difficulty in recognizing the internal opening and the course of the fistula tract can make successful surgery sometimes challenging [8].

Various radiological modalities were applied for evaluation of fistula patients; conventional fistulography was used [9] but its diagnostic yield is limited secondary to its difficulty to recognize the internal opening especially if blocked by debris [10]. Endosonography with color Doppler has greater diagnostic value than conventional grayscale endosonography for PAF evaluation [11]. 


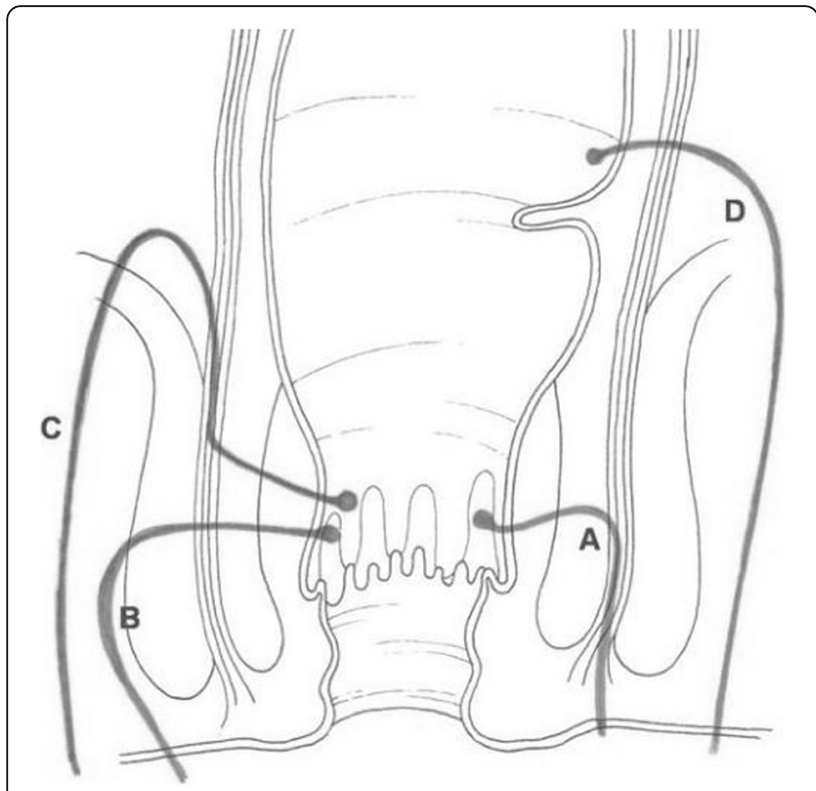

Fig. 1 Different types of perianal fistula according to Parks Classification. a Inter-sphincteric fistula. b Trans-sphincteric fistula. c Supra-sphincteric fistula. d Extra-sphincteric fistula

Three-dimensional ultrasonography (3D US) improves PAF detection and characterization, so it plays a crucial role in optimal treatment planning, but proficiency is one of its limitations [12]. Transperineal US is a very accurate diagnostic method, and for its simplicity and low cost, it is recommended to be the first diagnostic modality for anal fistula [13].

MRI is the reference standard for assessment of perianal fistula, defining anatomy and guiding surgery [14].
However, MRI technique is critical especially the selection of sequences for diagnosis and characterization of abnormalities with the main choices being between standard anatomical sequences (T1 or T2), assessing for edema (T2/fat-saturated MRI image (T2/FS) or short tau inversion recovery (STIR)), assessing abnormal contrast enhancement (T1/fat-saturated MRI image (T1/FS) post-contrast), and assessing for abnormal diffusion or a combination of these [15]. Moreover, for complex perianal fistula, 3D reconstruction is required to improve surgical planning. Furthermore, an animation of MRI fistulography needs to be created for trans-sphincteric fistula tract with a cephalad extension in the intersphincteric space [14].

The activity of PAF before primary surgical interference may hamper successful surgical outcome, and so could be considered as an additional independent risk factor for surgical failure. The present study tried to determine the ability of preoperative MRI for preoperative evaluation of anal fistula regarding activity, tract identification, and the impact on surgical outcome.

\section{Methods}

This is retrospective study for patients who underwent preoperative MRI and surgical interference from April 2015 to Jan 2018. The study protocol was approved by the Local Ethical Committee. All patients who presented with clinically suspected PAF and signed fully informed written consent to undergo the appropriate surgical procedure for fistula management were included in the preliminary data evaluation. Exclusion criteria include patients omitted from MR imaging for any cause, who

Table 1 MRI-based Van Assche scoring system

\begin{tabular}{|c|c|c|c|c|}
\hline & Score parameters & Items & & Score \\
\hline \multirow[t]{9}{*}{ Local extension } & \multirow[t]{4}{*}{ Number of fistula tracts } & None & & 0 \\
\hline & & \multirow[t]{2}{*}{ Single } & Unbranched & 1 \\
\hline & & & Branched & 2 \\
\hline & & \multicolumn{2}{|c|}{ Multiple } & 3 \\
\hline & \multirow[t]{3}{*}{ Location } & \multicolumn{2}{|c|}{ Extra- or inter-sphincteric } & 1 \\
\hline & & \multicolumn{2}{|c|}{ Trans-sphincteric } & 2 \\
\hline & & \multicolumn{2}{|c|}{ Supra-sphincteric } & 3 \\
\hline & \multirow[t]{2}{*}{ Extension } & \multicolumn{2}{|c|}{ Infralevatoric } & 1 \\
\hline & & \multicolumn{2}{|c|}{ Supralevatoric } & 2 \\
\hline \multirow[t]{7}{*}{ Active inflammation } & \multirow[t]{3}{*}{ Hyperintensity on T2-weighted images } & \multicolumn{2}{|l|}{ Absent } & 0 \\
\hline & & \multicolumn{2}{|l|}{ Mild } & 4 \\
\hline & & \multicolumn{2}{|c|}{ Pronounced } & 8 \\
\hline & \multirow[t]{2}{*}{ Collections (cavities $>3 \mathrm{~mm}$ in diameter) } & \multicolumn{2}{|l|}{ Absent } & 0 \\
\hline & & \multicolumn{2}{|l|}{ Present } & 4 \\
\hline & \multirow[t]{2}{*}{ Rectal wall involvement } & \multicolumn{2}{|l|}{ Norma } & 0 \\
\hline & & \multicolumn{2}{|c|}{ Thickened } & 2 \\
\hline
\end{tabular}




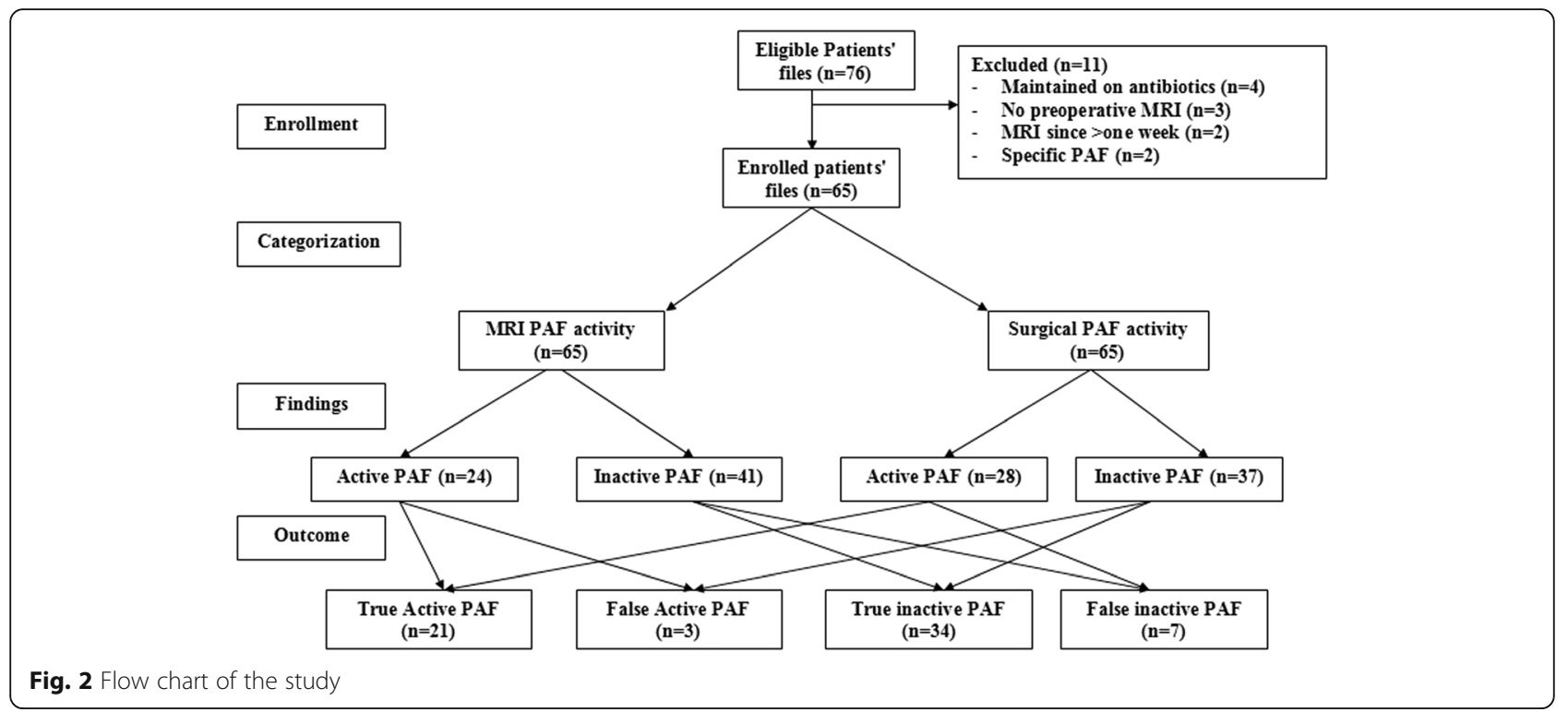

refused surgical interference, who received antibiotic therapy during the week before enrollment, maintained on anti-inflammatory drugs for any indication, and who did not undergo diffusion-weighted magnetic resonance imaging (DW-MRI), and patients who had surgical interference after more than 1 week of MR imaging were not enrolled in the study.

Extracted data included patient's demographic data including age, gender, weight, height, calculated body mass index, and disease-related data. Clinical data include presence of severe pain, restriction of daily and/or sexual activity, presence of inflamed skin, and discharge of pus. Fistula was clinically classified according to its relation to anal sphincters as inter-sphincteric, trans-sphincteric, supra-sphincteric, and extra-sphincteric fistula which correspond to Parks types 1-4, respectively [16] (Fig. 1).

MRI fistula imaging-related data were revised, and fistula severity was scored using criteria of both local extension of fistulas and active inflammation for a total maximum score of 22, according to the Van Assche scoring system (Table 1) which was primarily used in PAF with Crohn's disease [17].

\section{MRI protocol}

The applied MRI protocol was revised to assure of its competence with the study requirements. All patients received no bowel preparation, anal canal, or fistula catheterization. MRI was performed using a $1.5 \mathrm{~T}$ body MRI system (Magnetom Essenza, Siemens, Erlangen, Germany) and a pelvic phased-array coil. MRI protocol consisted of fat-suppressed non-contrast-enhanced T1weighted sequence (T1/FS) obtained in axial, coronal, and sagittal planes with repetition time/echo time (TR/ $\mathrm{TE}$ ) of $650 / 13 \mathrm{~ms}$, section thickness of $3 \mathrm{~mm}$, interslice gap of $0.75 \mathrm{~mm}$, matrix size of $384 \times 216$, field of view (FOV) of $320 \times 320 \mathrm{~mm}$, received bandwidth of 15.5 $\mathrm{kHz}$, and scan time of $4 \mathrm{~min}$ and $20 \mathrm{~s}$. A T2-weighted sequence was also obtained in the axial, coronal, and sagittal planes with TR/TE of $5600 / 95 \mathrm{~ms}$, section thickness of $3 \mathrm{~mm}$, interslice gap of $0.75 \mathrm{~mm}$, matrix size of $448 \times$ 336, FOV of $320 \times 320 \mathrm{~mm}$, received bandwidth of 19 $\mathrm{kHz}$, and scan time of $3 \mathrm{~min}$ and $49 \mathrm{~s}$. A STIR-weighted sequence was also obtained in the three planes with TR/ TE of $4410 / 33 \mathrm{~ms}$, section thickness of $3 \mathrm{~mm}$, interslice gap of $0.75 \mathrm{~mm}$, matrix size of $320 \times 332046$, FOV of $320 \times 270 \mathrm{~mm}$, received bandwidth of $16 \mathrm{kHz}$, and scan time of $4 \mathrm{~min}$ and $30 \mathrm{~s}$. The DW-MRI was obtained in axial plane with TR/TE of $6000 / 84.2 \mathrm{~ms}$, slice thickness of $4 \mathrm{~mm}$, interslice gap of $1 \mathrm{~mm}$, number of slices equals 24, matrix size of $192 \times 126$, FOV of $380 \times 309 \mathrm{~mm}$, bandwidth of $124 \mathrm{kHz}$, number of signal averages 4 , bvalues of 50,400 , and $800 \mathrm{~s} / \mathrm{mm}^{2}$, scan time of $2 \mathrm{~min}$ and $50 \mathrm{~s}$. Apparent diffusion coefficient (ADC) values of the fistulas were measured in axial DW-MRI (b-value = $50 \mathrm{~s} / \mathrm{m}^{2}$ ). Circular region of interest (ROI) area was placed on the largest suspected area, and the mean ADC value and its standard deviation (SD) were measured.

\section{Study outcome}

- Primary outcome included intraoperative fistula activity determined after anesthesia on surgical exploration prior to any manipulations.

Intraoperative (IO) detection of pus was considered as an indication for active fistulas, while absence of pus indicated inactive fistulas. The frequency of MRI detection of fistula activity was compared versus IO findings. 
Table 2 Patient's demographic and clinical data

\begin{tabular}{|c|c|c|c|}
\hline \multicolumn{3}{|l|}{ Data } & Finding \\
\hline \multirow[t]{6}{*}{ Age (years) } & \multicolumn{2}{|l|}{$<30$} & $11(16.9 \%)$ \\
\hline & \multicolumn{2}{|l|}{$30-39$} & $14(21.5 \%)$ \\
\hline & \multicolumn{2}{|l|}{$40-49$} & $20(30.8 \%)$ \\
\hline & \multicolumn{2}{|l|}{$50-59$} & $17(26.2 \%)$ \\
\hline & \multicolumn{2}{|l|}{$\geq 60$} & $3(4.6 \%)$ \\
\hline & \multicolumn{2}{|l|}{ Mean $( \pm S D)$} & $44.3 \pm 11.7$ \\
\hline \multirow[t]{2}{*}{ Gender } & \multicolumn{2}{|l|}{ Males } & $53(81.5 \%)$ \\
\hline & \multicolumn{2}{|l|}{ Females } & $12(18.5 \%)$ \\
\hline \multirow[t]{3}{*}{ Body mass index data } & \multicolumn{2}{|l|}{ Weight (kg) } & $79.3 \pm 11.2$ \\
\hline & \multicolumn{2}{|l|}{ Height (cm) } & $169.2 \pm 2.5$ \\
\hline & \multicolumn{2}{|l|}{ BMI $\left(\mathrm{kg} / \mathrm{m}^{2}\right)$} & $27.7 \pm 3.9$ \\
\hline \multirow[t]{6}{*}{ Elapsed time between presentation and MR imaging (days) } & \multicolumn{2}{|l|}{2} & $16(24.6 \%)$ \\
\hline & \multicolumn{2}{|l|}{3} & $9(13.8 \%)$ \\
\hline & \multicolumn{2}{|l|}{4} & $21(32.3 \%)$ \\
\hline & \multicolumn{2}{|l|}{5} & $13(20 \%)$ \\
\hline & \multicolumn{2}{|l|}{6} & $6(9.3 \%)$ \\
\hline & \multicolumn{2}{|l|}{ Mean $( \pm S D)$} & $4 \pm 1.3(2-6)$ \\
\hline \multirow[t]{4}{*}{ Pain } & \multicolumn{2}{|l|}{ No } & $10(15.4 \%)$ \\
\hline & \multicolumn{2}{|l|}{ Mild } & $18(27.7 \%)$ \\
\hline & \multicolumn{2}{|l|}{ Moderate } & $28(43 \%)$ \\
\hline & \multicolumn{2}{|l|}{ Severe } & $9(13.9 \%)$ \\
\hline \multirow[t]{4}{*}{ Effect on quality of life } & \multirow[t]{2}{*}{ Restriction of daily activities } & Yes & $12(18.5 \%)$ \\
\hline & & No & $53(81.5 \%)$ \\
\hline & Restriction of sexual activities & Yes & $18(27.7 \%)$ \\
\hline & & No & $47(72.3 \%)$ \\
\hline Perianal skin & No skin affection & & $43(66.2 \%)$ \\
\hline & Reddish and inflamed & & $16(24.6 \%)$ \\
\hline & Excoriated & & $6(9.2 \%)$ \\
\hline Pus discharge & No pus & & $47(72.3 \%)$ \\
\hline & Pus extruded from External orifice & Spontaneous & $4(6.2 \%)$ \\
\hline & & On pressure during examination & $7(10.8 \%)$ \\
\hline & Pus extruded from anus & Spontaneous & $2(3.1 \%)$ \\
\hline & & On pressure during examination & $5(4.6 \%)$ \\
\hline C-reactive protein level (mg/l) & $<5$ & & $59(90.8 \%)$ \\
\hline & $>5$ & & 6 (9.2\%) \\
\hline
\end{tabular}

Data are presented as mean \pm SD (standard deviation), numbers, and percentages

- Secondary outcome included the diagnostic validity characters of preoperative MRI for defining fistula activity.

\section{Statistical analysis}

Obtained data were presented as mean $\pm \mathrm{SD}$, ranges, numbers, and ratios. Numbers and ratios were analyzed using chi-square test ( $X^{2}$ test). Diagnostic validity characters included sensitivity and specificity rates and positive and negative predictive values (PPV and NPV). Sensitivity and PPV (=1 - specificity) of preoperative MRI as an indicator for fistula activity were analyzed using receiver operating characteristic curve (ROC) as judged by area under curve (AUC). The diagnostic significance of the determined AUC was compared to the null hypothesis that AUC $=0.500$ which is the area under the diagonal reference line. Statistical analysis was conducted using the IBM SPSS Statistics, Version 23, 
Table $3 \mathrm{MRI}$ findings according to Van Assche scoring system

\begin{tabular}{|c|c|c|c|c|}
\hline & Score parameters & Items ( & & Number $(\%$ \\
\hline \multirow[t]{10}{*}{ Local extension } & \multirow[t]{4}{*}{ Number of fistula tracts } & None & & $9(13.8 \%)$ \\
\hline & & \multirow[t]{2}{*}{ Single } & Unbranched (1) & $46(70.8 \%)$ \\
\hline & & & Branched (2) & $10(15.4 \%)$ \\
\hline & & \multicolumn{2}{|c|}{ Multiple (3) } & $0(0 \%)$ \\
\hline & \multirow[t]{4}{*}{ Location } & \multicolumn{2}{|c|}{ Extra-sphincteric (1) } & $28(43.1 \%)$ \\
\hline & & \multicolumn{2}{|c|}{ Inter-sphincteric (1) } & $25(38.5 \%)$ \\
\hline & & \multicolumn{2}{|c|}{ Trans-sphincteric (2) } & $9(13.8 \%)$ \\
\hline & & \multicolumn{2}{|c|}{ Supra-sphincteric (3) } & $3(4.6 \%)$ \\
\hline & \multirow[t]{2}{*}{ Extension } & \multicolumn{2}{|c|}{ Infralevatoric (1) } & $62(95.4 \%)$ \\
\hline & & \multicolumn{2}{|c|}{ Supralevatoric (2) } & $3(4.5 \%)$ \\
\hline \multirow[t]{7}{*}{ Active inflammation } & \multirow[t]{3}{*}{ Hyperintensity on T2-weighted images } & \multicolumn{2}{|c|}{ Absent (0) } & $21(32.3 \%)$ \\
\hline & & \multicolumn{2}{|c|}{ Mild (4) } & $25(38.5 \%)$ \\
\hline & & \multicolumn{2}{|c|}{ Pronounced (8) } & $19(29.2 \%)$ \\
\hline & \multirow[t]{2}{*}{ Collections (cavities > $3 \mathrm{~mm}$ in diameter) } & \multicolumn{2}{|c|}{ Absent (0) } & $41(63.1 \%)$ \\
\hline & & \multicolumn{2}{|c|}{ Present (4) } & $24(36.9 \%)$ \\
\hline & \multirow[t]{2}{*}{ Rectal wall involvement } & \multicolumn{2}{|c|}{ Normal (0) } & $56(86.2 \%)$ \\
\hline & & \multicolumn{2}{|c|}{ Thickened (2) } & $9(13.8 \%)$ \\
\hline
\end{tabular}

2015 (IBM Corp. Armonk, NY, USA) for Windows statistical package. $P$ value $<0.05$ was considered statistically significant.

\section{Results}

Seventy-six patients were eligible for evaluation, 11 were excluded and 65 patients were included in the study (Fig. 2), and extracted data are shown in Table 2.

Considering identification of internal fistula opening is mandatory to define fistula tract; otherwise, it is considered as pouch, and MRI defined 9 patients having pouches without definite internal opening, while the remaining 56 patients had definite internal opening; 10 patients had branched and 46 patients had unbranched fistula. During surgical exploration, the presence of internal opening was confirmed in 59 patients; 56 previously defined by MRI giving a true positive rate (TPR) of 100\%, 3 of those pouches defined by MRI were having internal openings, the true negative rate of $(66.7 \%)$, and total internal fistula definition rate of $95.4 \%$ (Table 3).

Median value of scoring of MRI findings was 8 (95\% CI $7-11$ ), and 36 studies were scored $\leq 8$, while 29 studies were scored $>8$. Median value of scoring of MRI findings concerning local extension was 3 (95\% CI 3-4), and concerning presence of active inflammation was 4 (95\% CI 4-8). Parks clinical grading showed positive significant correlation $(r=0.271, p=0.029)$ with MRI findings concerning local extension.

MRI finding concerning active inflammation showed presence of collections with cavities $>3 \mathrm{~mm}$ in diameter in 24 patients $(36.9 \%)$ and no collection in 41 patients (63.1\%) (Table 3). Surgical exploration documented the presence of collections and assured MRI findings in $21 \mathrm{pa}-$ tients (true positive cases) and absence of collections in 34 patients (true negative cases), while detected collections in 7 patients diagnosed on MRI as free of collection (false negative cases). Moreover, surgical exploration denied the presence of collection in three patients previously defined

Table 4 Visibility of PAF using different MRI pulse sequences

\begin{tabular}{|c|c|c|c|c|}
\hline MRI pulse sequences & No. of positive visible fistula & Sensitivity & Specificity & Accuracy \\
\hline T1 post-contrast & $54(83.1 \%)$ & $71.2 \%$ & $33.3 \%$ & $67.7 \%$ \\
\hline STIR & 55 (84.6\%) & $78 \%$ & $66.7 \%$ & $76.9 \%$ \\
\hline DW-MRI & 59 (90.8\%) & $83.1 \%$ & $100 \%$ & $84.6 \%$ \\
\hline DW-MRI + T1 post-contrast & 60 (92.3\%) & $84.8 \%$ & $100 \%$ & $86.2 \%$ \\
\hline DW-MRI + STIR & $61(93.8 \%)$ & $88.1 \%$ & $100 \%$ & $89.2 \%$ \\
\hline All sequences & $62(95.4 \%)$ & $88.7 \%$ & $100 \%$ & $90.1 \%$ \\
\hline
\end{tabular}


Table 5 Detection of collections defined as cavities of $>3 \mathrm{~mm}$ in diameter using different MRI pulse sequences

\begin{tabular}{|c|c|c|c|c|c|c|c|}
\hline \multirow[t]{2}{*}{ MRI pulse sequences } & \multicolumn{2}{|c|}{ Positive } & \multicolumn{2}{|c|}{ Negative } & \multirow[t]{2}{*}{ Sensitivity } & \multirow[t]{2}{*}{ Specificity } & \multirow[t]{2}{*}{ Accurac } \\
\hline & True & False & True & False & & & \\
\hline $\mathrm{T} 1$ post-contrast & 11 & 6 & 38 & 10 & $52.4 \%$ & $86.4 \%$ & $75.4 \%$ \\
\hline STIR & 13 & 6 & 38 & 8 & $61.9 \%$ & $86.4 \%$ & $78.5 \%$ \\
\hline DW-MRI & 17 & 5 & 39 & 4 & $81 \%$ & $88.6 \%$ & $86.2 \%$ \\
\hline DW-MRI + T1 post-contrast & 18 & 5 & 39 & 3 & $85.7 \%$ & $88.6 \%$ & $87.7 \%$ \\
\hline DW-MRI + STIR & 20 & 4 & 40 & 1 & $95.2 \%$ & $91 \%$ & $92.3 \%$ \\
\hline All sequences & 21 & 3 & 41 & 0 & $100 \%$ & $93.2 \%$ & $95.4 \%$ \\
\hline
\end{tabular}

by MRI as having perianal collection (false positive cases) (Fig. 2). Thus, preoperative MRI could predict the severity of perianal disease on surgical exploration with sensitivity, specificity, and accuracy rates of $75 \%, 92 \%$, and $84.6 \%$, respectively, and positive and negative likelihood ratios of 9.25 (95\% CI 3.06-27.95) and 0.27 (95\% CI 0.14-0.52), respectively. Moreover, surgical findings concerning disease severity showed positive significant correlation $(r=0.369$, $p=0.002$ ) with MRI findings.

Comparison of MRI pulse sequences as regards the ability of each sequence for fistula visualization showed that DW-MRI provided the highest sensitivity and accuracy for fistula visualization with $100 \%$ specificity rate and significant difference versus $\mathrm{T} 1$ post-contrast sequence. Moreover, addition of DW-MRI to each of T1 post-contrast and STIR sequences improved sensitivity and accuracy of fistula visualization without affecting its specificity (Table 4).

Differentially, all MRI pulse sequences showed high specificity and accuracy for detection of collections defined as cavities of $>3 \mathrm{~mm}$ in diameter, but their sensitivity was variable as being lowest with $\mathrm{T} 1$ post-contrast and highest with DW-MRI. Combined imaging using DW-MRI sequence with either T1 post-contrast or STIR sequences improved the sensitivity and accuracy rate of both for diagnosis of acute inflammation, but the effect was more pronounced with STIR sequence. Interestingly, using the three sequences allowed sensitivity to approach $100 \%$ with higher specificity and accuracy than any single sequence or combination of sequences (Table 5).

The ROC curve analysis for differentiation between the three MRI pulse sequences as regards the ability of each sequence for fistula visualization showed that DWMRI provided the highest specificity rate $(\mathrm{AUC}=0.848$, $p=0.0006)$, followed by STIR (AUC $=0.741, p=0.002$ ), and lastly $\mathrm{T} 1$ post-contrast (AUC $=0.694, p=0.012$ ) sequences (Fig. 3). Concerning the combinations, ROC curve analysis found addition of DW-MRI to either of STIR (AUC $=0.931, p=0.0004$ ) and T1 post-contrast (AUC $=0.872, p=0.0007$ ) sequences improved specificity (Fig. 4). Regression analysis defined DW-MRI as the single significant sequence $(\beta=0.688, p=0.0009)$ and the combination of STIR and DW-MRI $(\beta=0.835, p=$ $0.0003)$ as the significant combined sequences for detection of acute inflammation (Table 5).

\section{Discussion}

Through the duration of the study, MRI could detect the fistula internal opening by a rate of $95.4 \%$ in comparison to surgical findings with $100 \%$ true positive detection rate. Moreover, preoperative MRI changed surgical decision by a rate of $16.9 \%$. Median score of MRI findings was 8 , for local extension was 3 , and 4 for presence of active inflammation with a positive significant correlation with Parks clinical grading.

In line with these findings, Singh et al. [18] reported high sensitivity of MRI in identification of secondary tract, correct localization of internal opening, and correct detection of horse-shoeing with rates of $93.75 \%$, $95.83 \%$, and $87.5 \%$, respectively. Also, Chauhan et al. [19] reported that MRI showed a high sensitivity rate of 93.7\% and positive predictive value (PPV) of $96.7 \%$ when correlated with surgical findings. Recently, Karanikas et al. [20] reported a success rate of $77.4 \%$ to demonstrate the presence of a fistula in their series

DW-MRI, in comparison to the other pulse sequences, provided high sensitivity and accuracy with $100 \%$ specificity for fistula visualization and highest sensitivity, specificity, and accuracy for detection of cavities $>3 \mathrm{~mm}$ in diameter. Similarly, Singh et al. [18] found the sensitivity and specificity of MRI in correctly detecting and grading the primary tract at $95.56 \%$ and $80 \%$, respectively; for abscess, it was $87.50 \%$ and $95.24 \%$, respectively. Yoshizako et al. [21] reported sensitivity of $95.7 \%$ and negative predictive value (NPV) of $90 \%$ for DW-MRI at optimal cutoff apparent diffusion coefficient (ADC) of 1.109 for diagnosing fistula activity. Also, Dohan et al. [22] reported a sensitivity rate of $100 \%$ for DW-MRI and 91.2 $\%$ for T2-weighted MRI for fistula detection.

The addition of DW-MRI to either T1 post-contrast or STIR sequence improved sensitivity and accuracy of fistula visualization without affecting specificity of DWMRI, while for detection of cavities of $>3 \mathrm{~mm}$ in diameter using the three sequences allowed sensitivity to 


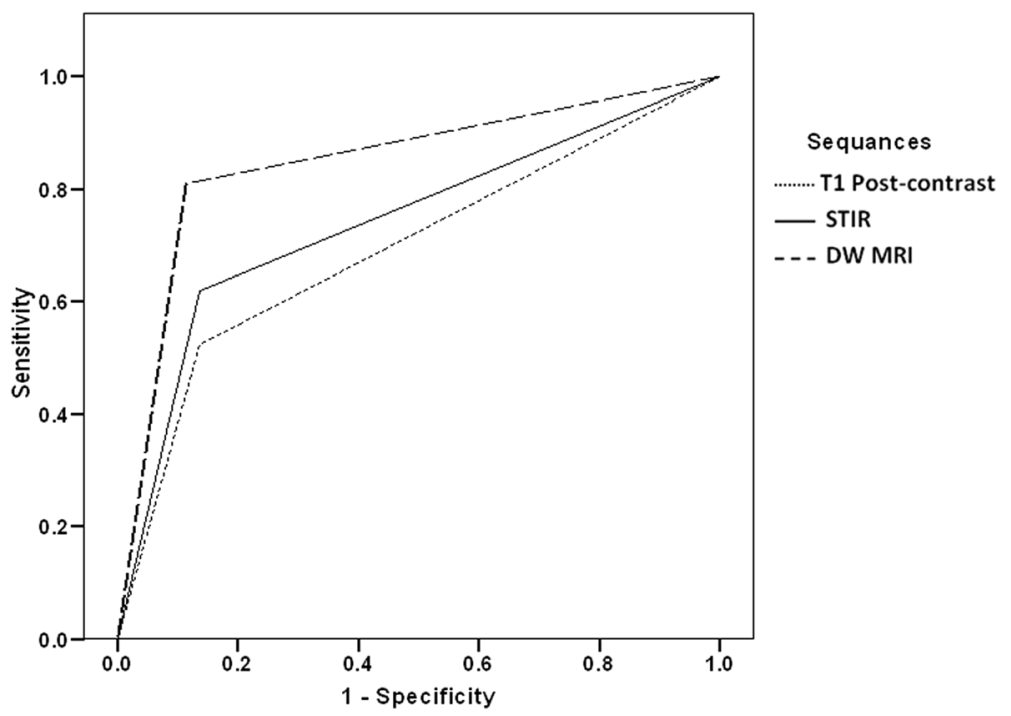

Fig. 3 ROC curve analysis of MRI sequences for identification of active inflammation of the fistula

approach $100 \%$ with higher specificity and accuracy than any single sequence.

These findings go in hand with Dohan et al. [22] who reported an area under the ROC curve of 0.971 with a sensitivity of $100 \%$, a specificity of $90 \%$, PPV of $93 \%$, and NPV of $90 \%$ for an abscess diagnosis. Also, Cavusoglu et al. [23] found the confidence scores for perianal fistula diagnosis using combination of gadolinium chelate-enhanced T1 and T2-WI were greater than T2$\mathrm{WI}$ alone and confidence scores using the combination of DW-MRI and T2-WI were greater than T2-WI alone with greater sensitivity and specificity.
ROC curve analysis showed that DW-MRI provided the highest specificity rate among the three MRI pulse sequences for fistula visualization, and its addition to any of other sequences improved specificity. Also, regression analysis defined DW-MRI as the single significant sequence and the combination of DW-MRI and STIR images as the significant combined sequences for detection of acute inflammation.

These findings coincide with Singh et al. [18] who documented that MRI was highly accurate in assessment of surgically important parameters of perianal fistulae and imaging findings on T2-WI and post-contrast T1-

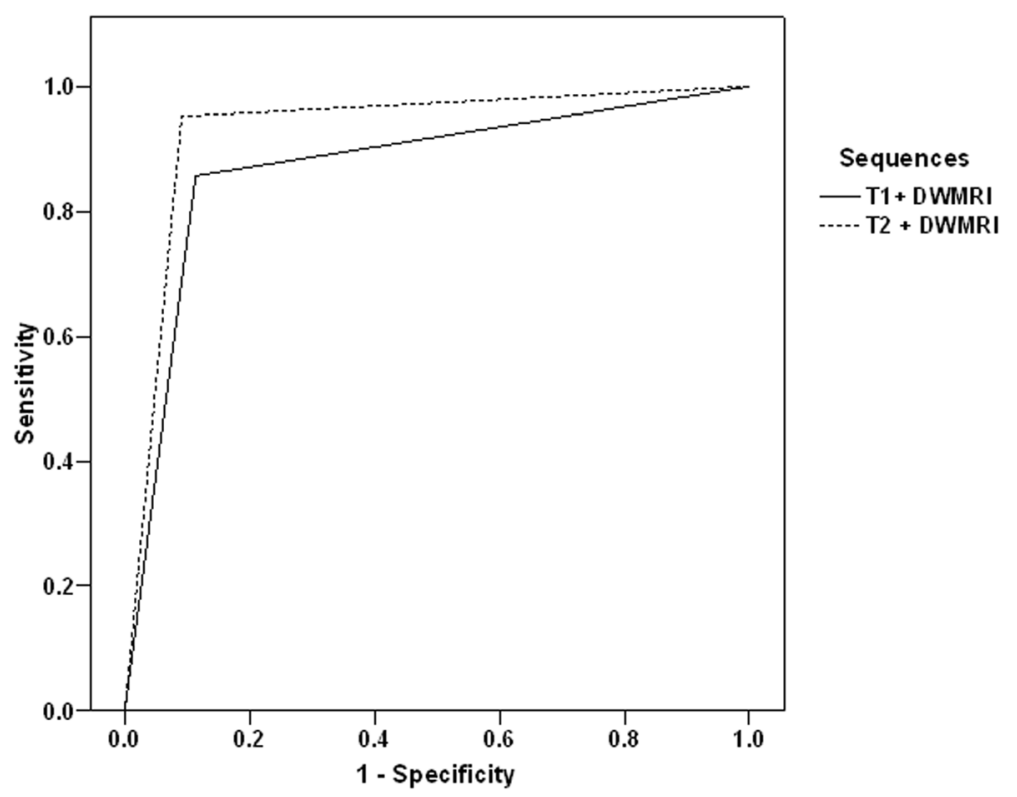

Fig. 4 ROC curve analysis of combinations of MRI sequences for identification of active inflammation of the fistula 

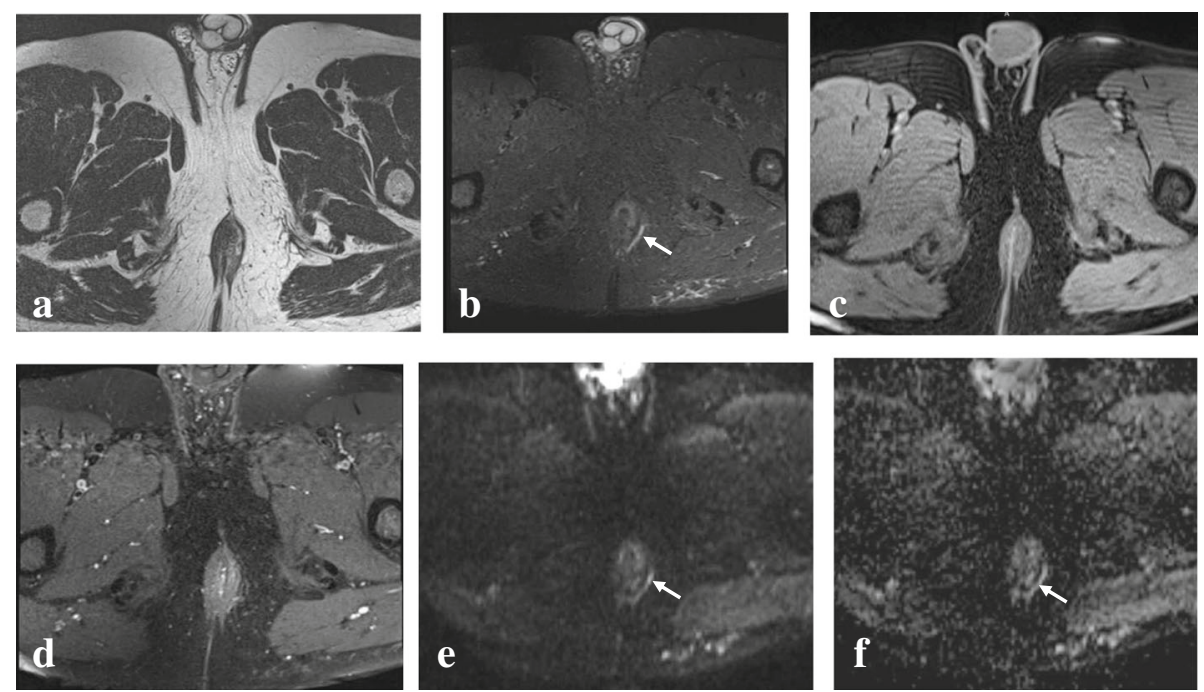

Fig. 5 A 38-year-old man with left inter-sphincteric fistula without abscess. a Axial T2-weighted image, the perianal fistula is not visualized. b Axial STIR-weighted image reveals high signal intensity left perianal fistula (arrow). c Axial T1 fat-saturated weighted image, the perianal fistula is not visualized. d Axial T1 fat-saturated post-contrast injection weighted image, the perianal fistula is not visualized. e Axial diffusion-weighted image reveals high signal intensity of the fistula (arrow). $\mathbf{f}$ Axial ADC mapping shows high signal intensity of the fistula (arrow)

weighted fat-saturated sequences were statistically similar, so recommended to omit contrast study. Then, Yoshizako et al. [21] who assessed the feasibility of using DW-MRI for evaluating perianal fistula activity documented that DW-MRI is a feasible method for evaluating perianal fistula activity with area under the ROC curve of 0.749 .

Thereafter, Cavusoglu et al. [23] documented that DW-MRI has a significant added value compared to T2WI alone in the diagnosis of perianal fistula and Baik et al. [24] found combined DW-MRI and CE-T1WI might improve fistula conspicuity with comparable diagnostic performance to T2WI. Recently, Wang et al. [25] reported that fractional anisotropy (FA) and ADC values for positive inflammation activity were significantly lower than those for negative inflammatory activity and concluded that FA and ADC values were instrumental in evaluating the activity of perianal fistulas.

In support of the efficacy of MRI for perianal fistula evaluation, Lee et al. [26] used MRI as a comparative gold standard and found transperitoneal US corresponded with MRI findings with sensitivity and PPV of
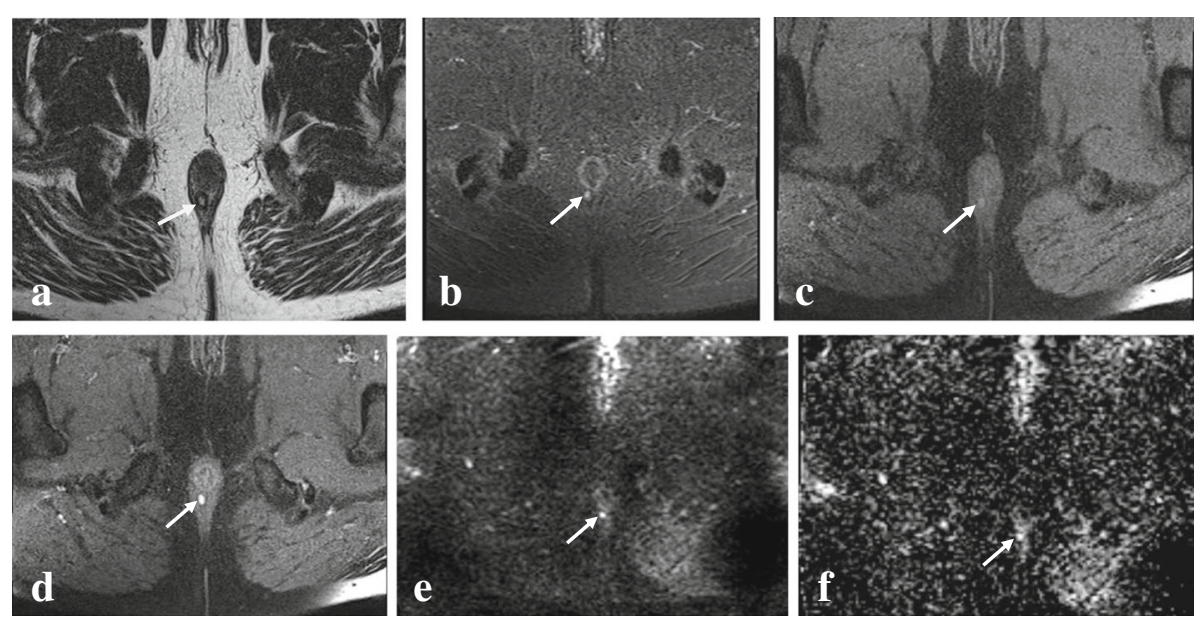

Fig. 6 A 51-year-old man with inter-sphincteric fistula without abscess. a Axial T2-weighted image shows a perianal fistula (arrow) that exhibits high signal intensity. b Axial STIR-weighted image reveals high signal intensity of the fistula (arrow). c Axial T1 fat-saturated weighted image reveals intermediate signal intensity of the fistula (arrow). $\mathbf{d}$ Axial T1 fat-saturated post-contrast injection weighted image reveals intense contrast enhancement of the fistula (arrow). e Axial diffusion-weighted image reveals high signal intensity of the fistula (arrow). $\mathbf{f}$ Axial ADC mapping reveals high signal intensity of the fistula (arrow) 

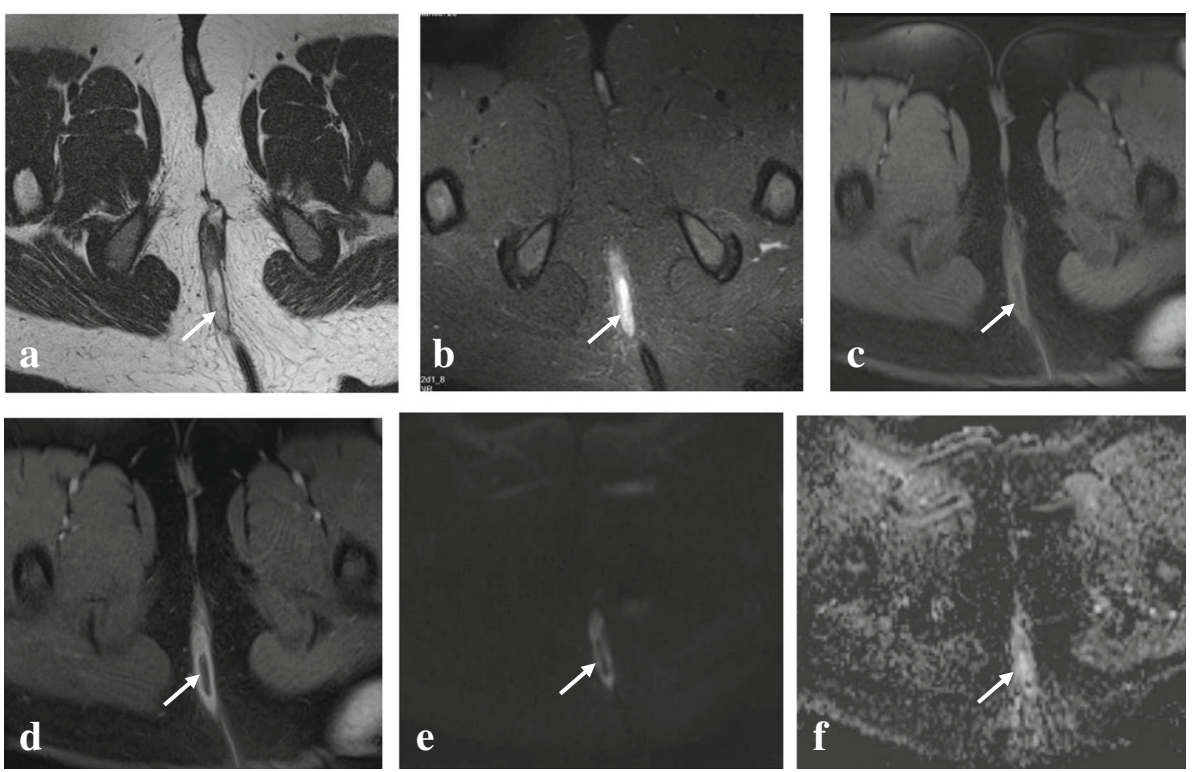

Fig. 7 A 17-year-old boy with perineal abscess seen at 6 o'clock of the anal canal opens through an inter-sphincteric sinus tract to the anus. a Axial T2-weighted image shows a perineal abscess (arrow) that exhibits high signal intensity. b Axial STIR-weighted image reveals high signal intensity of the perineal abscess (arrow). c Axial T1 fat-saturated weighted image reveals intermediate signal intensity of the perineal abscess (arrow). d Axial T1 fat-saturated post-contrast injection weighted image reveals contrast enhancement of the perineal abscess walls (arrow). e Axial diffusion-weighted image reveals high signal intensity of the perineal abscess walls (arrow). $\mathbf{f}$ Axial ADC mapping shows high signal intensity of the perineal abscess

$76.3 \%$ and $84.2 \%$ for fistula detection and $56.3 \%$ and $90.0 \%$ for diagnosis of abscess cavity, while colonoscopy corresponded with MRI findings with sensitivity and PPV of $67.8 \%$ and $89.9 \%$, respectively, for fistula detection and $43.8 \%$ and $48.8 \%$ for abscess detection.

\section{Conclusion}

MRI is a valuable and accurate preoperative investigation for evaluation of perianal fistula activity and abscess localization, so it can aid surgical decision making. Moreover, MRI allowed accurate fistula detection,
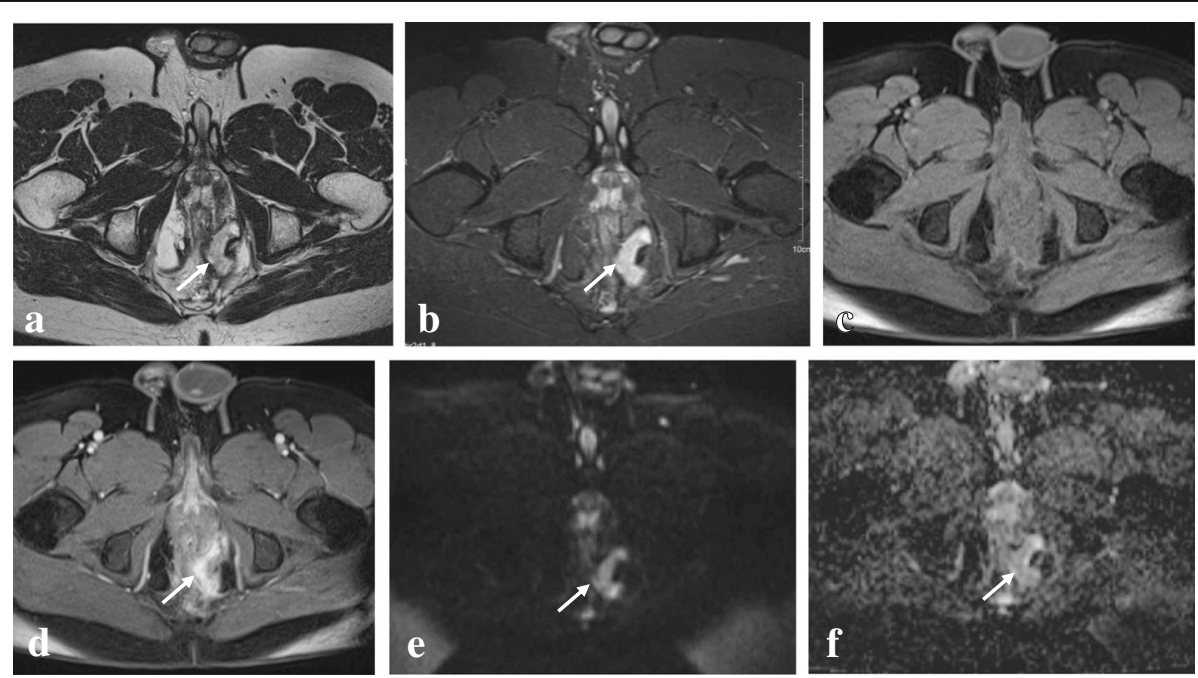

Fig. 8 A 26-year-old man with supra-sphincteric fistula with abscess. a Axial T2-weighted image shows C-shaped left perianal abscess (arrow) that exhibits high signal intensity. b Axial STIR-weighted image reveals high signal intensity of the perianal abscess (arrow). c Axial T1 fat-saturated weighted image the perianal abscess is not visualized. $\mathbf{d}$ Axial T1 fat-saturated post-contrast injection weighted image reveals intense contrast enhancement of the perianal abscess (arrow). e Axial diffusion-weighted image reveals high signal intensity of the perianal abscess (arrow). $\mathbf{f}$ Axial ADC mapping shows high signal intensity of the perianal abscess 
internal opening identification, and evaluation of its relation to sphincters, so it can help surgical procedure planning. DW-MRI is a valuable sequence with highest diagnostic yield, and its addition to STIR and T1 postcontrast images improves sensitivity and specificity for determination of fistula activity and extension (Figs. 5, 6, 7 , and 8).

\section{Abbreviations}

PAF: Perianal fistula; MRI: Magnetic resonance imaging; DW-MRI: Diffusionweighted magnetic resonance imaging; ADC: Apparent diffusion coefficient; STIR: Short tau inversion recovery; PDAl: Perianal Disease Activity Index; 3D US: Three-dimensional ultrasonography; T2/FS: T2/fat-saturated MRI image; T1/FS: T1/fat-saturated MRI image; IO: Intraoperative; CE-T1WI: Contrastenhanced T1 weighted image; FA: Fractional anisotropy

\section{Acknowledgements}

Not applicable.

\section{Authors' contributions}

HYA and DBE suggested and developed the research idea, reviewed the literature, did the data collection and analysis, performed the statistical analysis, wrote and revised the manuscript, and prepared the MRI cases, figures, and tables. MAB followed the surgical findings with analysis of operative data and did the manuscript revision. NAZ followed the surgical findings with analysis of operative data and did the manuscript revision. All authors read and approved the final manuscript.

\section{Funding}

This study had no funding from any resource.

\section{Availability of data and materials}

The datasets used and/or analyzed during the current study are available from the corresponding author on reasonable request.

\section{Ethics approval and consent to participate}

This study was approved by Benha University-Faculty of medicine-Research Ethics Committee (BFOM-REC)-Egypt. The reference number is not applicable. Written informed consent was obtained from all patients. If the patient was less than 16 years old or unconscious at the time of the study, written informed consent for their participation was given by their parent or legal guardian.

\section{Consent for publication}

All patients included in this research gave written informed consent to publish the data contained within this study. If the patient was less than 16 years old, deceased, or unconscious when consent for publication was requested, written informed consent for the publication of this data was given by their parent or legal guardian.

\section{Competing interests}

The authors declare that they have no competing interests.

\section{Author details}

${ }^{1}$ Radiodiagnosis Department, Faculty of Medicine, Benha University, Benha, Egypt. ${ }^{2}$ General Surgery Department, Faculty of Medicine, Menoufia University, Shibin El Kom, Egypt. ${ }^{3}$ General Surgery Department, Faculty of Medicine, Benha University, Benha, Egypt.

Received: 21 November 2019 Accepted: 26 November 2019

Published online: 10 December 2019

\section{References}

1. Seow-Choen F, Nicholls RJ (1992) Anal fistula. Br J Surg. 79(3):197-205

2. Igwe PO, Dodiyi-Manuel A, Oparaku KC (2014) The pattern of surgically treatable anorectal diseases in University of Port Harcourt Teaching Hospital, Rivers State, Nigeria. Niger J Med. 23(1):57-60
3. Felt-Bersma RJ, Bartelsman JF (2009) Haemorrhoids, rectal prolapse, anal fissure, peri-anal fistulae and sexually transmitted diseases. Best Pract Res Clin Gastroenterol. 23(4):575-592

4. Abeysuriya V, Salgado LS, Samarasekera DN (2010) The distribution of the anal glands and the variable regional occurrence of fistula-in-ano: is there a relationship? Tech Coloproctol. 14(4):317-321

5. Sahnan K, Askari A, Adegbola SO, Tozer PJ, Phillips RKS, Hart A, Faiz OD (2017) Natural history of anorectal sepsis. Br J Surg. 104(13):1857-1865

6. Schäfer AO (2018) Perianal inflammatory diseases: classification and imaging. Radiologe. 58(4):344-354

7. Cafaro D, Onofrio L (2006) Transrectal ultrasonography by rotating feeler in the perianal fistulae/abscesses surgery. Anatomo-functional description. Ann Ital Chir. 77(4):369-375

8. Jayarajah U, Samarasekera DN (2017) Predictive accuracy of Goodsall's rule for fistula-in-ano. Ceylon Med J. 62(2):97-99

9. Pomerri F, Pittarello F, Dodi G, Pianon P, Muzzio PC (1988) Radiologic diagnosis of anal fistulae with radio-opaque markers. Radiol Med. 75(6):632-637

10. D'Hoore A, Penninckx F (2000) The pathology of complex fistula in ano. Acta Chir Belg. 100(3):111-114

11. Di Nardo R, Drudi FM, Marziale P, lannicelli E, Righi A, Trippa F, Cascone F, Passariello R (2000) Role of color Doppler echography in the visualization of perianal fistulae with injections of physiologic solutions. Radiol Med 100(4):235-239

12. Kim MJ (2015) Transrectal ultrasonography of anorectal diseases: advantages and disadvantages. Ultrasonography. 34(1):19-31

13. Bor R, Farkas K, Bálint A, Szűcs M, Ábrahám S, Milassin Á, Rutka M, Nagy F, Milassin P, Szepes Z, Molnár T (2016) Prospective comparison of magnetic resonance imaging, transrectal and transperineal sonography, and surgical findings in complicated perianal Crohn disease. J Ultrasound Med. 35(11):2367-2372

14. Sahnan K, Adegbola SO, Tozer PJ, Patel U, llangovan R, Warusavitarne J, Faiz OD, Hart AL, Phillips RKS, Lung PFC (2018) Innovation in the imaging perianal fistula: a step towards personalised medicine. Therap Adv Gastroenterol. 11:1756284818775060

15. Tolan DJ (2016) Magnetic resonance imaging for perianal fistula. Semin Ultrasound CT MR. 37(4):313-322

16. Parks AG, Gordon PH, Hardcastle JD (1976) A classification of fistula-in-ano. $\mathrm{Br} J$ Surg. 63(1):1-12

17. Van Assche G, Vanbeckevoort D, Bielen D, Coremans G, Aerden I, Noman M D'Hoore A, Penninckx F, Marchal G, Cornillie F, Rutgeerts P (2003) Magnetic resonance imaging of the effects of infliximab on perianal fistulizing Crohn's disease. Am J Gastroenterol. 98(2):332-339

18. Singh K, Singh N, Thukral C, Singh KP, Bhalla V (2014) Magnetic resonance imaging (MRI) evaluation of perianal fistulae with surgical correlation. J Clin Diagn Res. 8(6):RC01-RC04

19. Chauhan NS, Sood D, Shukla A (2016) Magnetic resonance imaging (MRI) characterization of perianal fistulous disease in a rural based tertiary hospital of North India. Pol J Radiol. 81:611-617

20. Karanikas I, Koutserimpas C, Siaperas P, Skarpas A, Karoubalis J, Velimezis G (2018) Transrectal ultrasonography of perianal fistulas: a single center experience from a surgeon's point of view. G Chir. 39(4):258-260

21. Yoshizako T, Wada A, Takahara T, Kwee TC, Nakamura M, Uchida K, Hara S, Luijten PR, Kitagaki H (2012) Diffusion-weighted MRI for evaluating perianal fistula activity: feasibility study. Eur J Radiol. 81(9):2049-2053

22. Dohan A, Eveno C, Oprea R, Pautrat K, Placé V, Pocard M, Hoeffel C, Boudiaf M, Soyer $P$ (2014) Diffusion-weighted MR imaging for the diagnosis of abscess complicating fistula-in-ano: preliminary experience. Eur Radiol. 24(11):2906-2915

23. Cavusoglu M, Duran S, Sözmen Cilız D, Tufan G, Hatipoglu Çetin HG, Ozsoy A, Sakman B (2017) Added value of diffusion-weighted magnetic resonance imaging for the diagnosis of perianal fistula. Diagn Interv Imaging. 98(5):401-408

24. Baik J, Kim SH, Lee Y, Yoon JH (2017) Comparison of T2-weighted imaging, diffusion-weighted imaging and contrast-enhanced T1-weighted MR imaging for evaluating perianal fistulas. Clin Imaging. 44:16-21

25. Wang Y, Gu C, Huo Y, Han W, Yu J, Ding C, Zhao X, Meng Y, Li C (2018) Diffusion tensor imaging for evaluating perianal fistula: feasibility study. Medicine (Baltimore). 97(29):e11570

26. Lee EH, Yang HR, Kim JY (2018) Comparison of transperianal ultrasound with colonoscopy and magnetic resonance imaging in perianal Crohn disease. J Pediatr Gastroenterol Nutr. 66(4):614-619

\section{Publisher's Note}

Springer Nature remains neutral with regard to jurisdictional claims in published maps and institutional affiliations. 